\title{
The Novel Fibrosis Index at Diagnosis May Predict All-cause Mortality in Patients With Antineutrophil Cytoplasmic Antibody-associated Vasculitis Without Substantial Liver Diseases.
}

\section{Jung Yoon Pyo}

Yonsei University College of Medicine

\section{Sung Soo Ahn}

Yonsei University College of Medicine

\section{Lucy Eunju Lee}

Yonsei University College of Medicine

\section{Gwang-mu Choi}

Yonsei University College of Medicine Jason Jungsik Song

Yonsei University College of Medicine

\section{Yong-Beom Park}

Yonsei University College of Medicine

\section{Sang-Won Lee ( $\nabla$ sangwonlee@yuhs.ac)}

Division of Rheumatology, Department of Internal Medicine, Yonsei University College of Medicine, Seoul, Republic of Korea https://orcid.org/0000-0002-8038-3341

Research article

Keywords: Antineutrophil cytoplasmic antibody-associated vasculitis, novel fibrosis index, predict, allcause mortality

Posted Date: August 27th, 2020

DOI: https://doi.org/10.21203/rs.3.rs-62370/v1

License: (1) This work is licensed under a Creative Commons Attribution 4.0 International License. Read Full License

Version of Record: A version of this preprint was published at Clinics on January 1st, 2021. See the published version at https://doi.org/10.6061/clinics/2021/e2501. 


\section{Abstract}

Objectives: Antineutrophil cytoplasmic antibody associated vasculitis (AAV) is a fatal disease. Currently, predictors of mortality of AAV are based on distribution of organ involvement. Novel fibrosis index (NFI) is an index, which is composed of laboratory results, that reflects the degree of liver fibrosis. The aim of this study is to evaluate whether NFI can predict poor outcomes among patients with AAV without substantial liver diseases.

Methods: 210 immunosuppressive drug-naïve AAV patients were retrospectively reviewed. NFI was calculated as follows: NFI= [serum bilirubin $x$ (alkaline phosphatase) ${ }^{2}$ ] / [platelet count $x$ (serum albumin $)^{2}$. Cut-off of NFI was set at 1.24, and patients were divided into two groups. Poor outcomes were defined as all-cause mortality, relapse, and end-stage renal disease (ESRD).

Results: During the median 34.5 months of follow up, twenty-one patients (10\%) died, 72 patients (34.3\%) relapsed, and 38 patients $(18.1 \%)$ reached ESRD due to AAV progression. The median calculated NFI was 0.61 , and it was higher in AAV patients with all-cause mortality than those without mortality, but it didn't reach the statistical significance (1.26 vs. 0.59 ). AAV patients with $\mathrm{NFI}$ at diagnosis $\geq 1.24$ exhibited a significantly lower cumulative patients' survival rate than those with NFI at diagnosis $<1.24(P=0.002)$. Multivariate Cox hazard model analysis showed that NFI at diagnosis $\geq 1.24$ was an independent predictor of all-cause mortality in AAV (HR $2.850,95 \% \mathrm{Cl} 1.026,7.910)$.

Conclusions: NFI at diagnosis $\geq 1.24$ may be an independent predictive marker for all-cause mortality in AAV patients without substantial liver diseases.

\section{Introduction}

Antineutrophil cytoplasmic antibody (ANCA)-associated vasculitis (AAV) is one of the systemic vasculitides and characterised by necrotising vasculitis in small-sized vessels including arterioles, venules and capillaries and occasionally arteries. AAV has three subtypes based on the histological and clinical features such as microscopic polyangiitis (MPA), granulomatosis with polyangiitis (GPA) and eosinophilic granulomatosis with polyangiitis (EGPA) [1]. So far various classification criteria and definitions have been suggested and currently, the 2007 European Medicine Agencies algorithm for AAV and the 2012 revised International Chapel Hill Consensus Conference Nomenclature of Vasculitides are widely used for the diagnosis of AAV [1, 2]. The disease activity of AAV is assessed and expressed by Birmingham vasculitis activity score (BVAS), which consists of nine items [3]. In addition, vasculitis damage index (VDI) is used to assess the irreversible damage caused by AAV and five-factor score (FFS) is used to predict the prognosis $[4,5]$.

The details of BVAS or VDI include several major organs, but they do not contain the symptoms or damages pertaining to liver involvement of AAV. In a similar context, a previous study reported that the progression of liver involvement or autoimmune hepatitis was rarely observed in AAV patients [6]. Meanwhile, other previous studies have reported that the indices for liver fibrosis were associated with all- 
cause mortality in patients AAV [7] and those with rheumatoid arthritis [8]. With regard to AAV, a hypothesis is more persuasive that these results might have resulted from the association of the variables, which compose the formulae of the indices for liver fibrosis, with the cross-sectional inflammatory burden and its related poor outcomes of AAV rather than the direct association between the indices for liver fibrosis and liver involvement of AAV.

Recently, two similar indices for liver fibrosis have been introduced such as fibrosis cirrhosis index (FCl) and novel fibrosis index (NFI). NFI is a modified formula by squaring both alkaline phosphatase in the molecule of FIC and serum albumin in the denominator of a formula of $\mathrm{FCl}$. That is, $\mathrm{NFI}$ is an amplification of the reflected contribution of alkaline phosphatase, which shows a positive correlation with liver fibrosis, and serum albumin, which shows a negative correlation [9]. Among the variables of NFI, there have been few previous reports on the association of AAV prognosis with bilirubin or alkaline phosphatase at diagnosis, but the association of AAV prognosis and either platelet count or serum albumin at diagnosis have already been demonstrated $[10,11]$. For these reasons, it can be reasonably speculated that NFI at diagnosis could predict the poor outcomes of AAV during follow-up in AAV patients. However, there has been no report on the clinical significance of NFI at diagnosis in the disease course of AAV to date. Hence, in this study, we investigated whether NFI at diagnosis might be associated with the cross-sectional activity of AAV and furthermore could predict the poor outcomes of AAV, particularly all-cause mortality, during follow-up in immunosuppressive drug-naïve patients AAV patients without substantial chronic liver diseases.

\section{Patients And Methods}

\section{Patients}

We retrospectively reviewed the medical records of 210 immunosuppressive drug-naïve AAV patients, who had been classified as AAV based on the 2007 EMA algorithm and the $2012 \mathrm{CHCC}$ definitions at the Division of Rheumatology, the Department of Internal Medicine, Yonsei University College of Medicine, Severance Hospital between October 2000 and March 2020 [1, 2]. BVAS and FFS were collected and if not available, they were calculated based on clinical and laboratory data documented in the medical records. Confirmation of ANCA both by an indirect immunofluorescence assay (IFA) for perinuclear (P)-ANCA and cytoplasmic (C)-ANCA and antigen-specific assays for myeloperoxidase (MPO)-ANCA and proteinase 3 (PR3)-ANCA was also included in the medical records. Patients negative by antigen-specific assay but positive for ANCA by IFA were considered to have MPO-ANCA or PR3-ANCA when AAV was strongly suspected by the clinical and laboratory features [12]. All patients included in this study had been followed up for at least 3 months or longer'. Patients had no serious medical conditions such as coexisting malignancies, serious infections and chronic liver diseases affecting the value of $\mathrm{NFI}$ at diagnosis. Also, they had never received immunosuppressive drugs prior to diagnosis. This study was approved by the Institutional Review Board of Severance Hospital (4-2017-0673). The need for patients' written informed consent was waived, as this was a retrospective study. 


\section{Exclusion substantial liver diseases}

Substantial liver diseases were identified by blood tests for liver function and viral hepatitis A, B and C and imaging studies such as computed tomography or ultrasonography at diagnosis. In addition, they were also confirmed by the 10th revised International Classification Diseases.

\section{Clinical data at diagnosis and during follow-up}

At diagnosis, age, gender, body mass index and smoking history were obtained. AAV subtypes, ANCA positivity, BVAS and FFS were also collected as AAV-specific indices. Chronic kidney disease (stage 3-5), diabetes mellitus, hypertension and interstitial pneumonia, were assessed. Erythrocyte sedimentation rate (ESR) and C-reactive protein (CRP) were also reviewed as acute-phase reactants. Medications administered during follow-up including glucocorticoid, cyclophosphamide, rituximab, azathioprine, mycophenolate mofetil, tacrolimus and methotrexate, were also counted.

\section{Poor outcomes}

All-cause mortality, relapse and end-stage renal disease (ESRD) were evaluated as the poor outcomes of AAV during follow-up. The follow-up period was defined as the duration between diagnosis and last visit for the survived patients. In cases of the deceased patients, it was defined as the duration between diagnosis and death. For patients who had relapse and ESRD, it was defined as the duration starting from diagnosis until relapse or the initiation of renal replacement therapy, respectively.

\section{Equation for $\mathrm{FCl}$ and $\mathrm{NFI}$}

$\mathrm{FCl}$ and NFI were calculated by the following formulae: $\mathrm{FCl}=$ (serum bilirubin $\mathrm{x}$ alkaline phosphatase) / (platelet count $x$ serum albumin), and NFI = [serum bilirubin $x$ (alkaline phosphatase $\left.)^{2}\right] /[$ platelet count $x$ (serum albumin) $\left.{ }^{2}\right]$

\section{Statistical analyses}

All statistical analyses were conducted using SPSS software (version 23 for Windows; IBM Corp., Armonk, NY, USA). Continuous variables were expressed as a median (interquartile range, IQR) and categorical variables were expressed as number and the percentage. Significant differences in categorical variables between the two groups were analysed using the Chi-square and Fisher's exact tests and among more than three groups using the ANOVA analysis. Significant differences in continuous variables between the two groups were compared using the Mann-Whitney test. The correlation coefficient between the two groups was obtained using the Pearson or the Spearman correlation analyses. Comparison of the cumulative survivals rates between the two groups was analysed by the Kaplan-Meier survival analysis with the log-rank test. The multivariable Cox hazards model analysis using variables with statistical significance in the univariable Cox hazards model analysis was conducted to appropriately obtain the hazard ratios (HRs) during the considerable the follow-up duration. The relative risk (RR) of A for $B$ was analysed using contingency tables and the chi square test. P-values less than 0.05 were considered statistically significant. 


\section{Results}

\section{Characteristics of AAV patients at diagnosis}

The median age of patients was 59.0 years and $32.5 \%$ were men. The median body mass index (BMI) was $22.2 \mathrm{~kg} / \mathrm{m} 2$ and only 7 patients had ever smoked but not current smokers. The most common AAV subtype and detected ANCA type were MPA (55.2\%) and MPO-ANCA (or P-ANCA) (66.7\%). The median BVAS and FFS were 12.0 and 1.0. The most common comorbidity was hypertension (46.7\%), followed by chronic kidney disease (stage 3-5) (28.6\%). The median ESR, CRP, NFI and FCl were $60.5 \mathrm{~mm} / \mathrm{hr}$, $13.2 \mathrm{mg} / \mathrm{L}, 0.61$ and 0.032 , respectively. Twenty-one patients (10\%) died, 72 patients (34.3\%) relapsed, and 38 patients $(18.1 \%)$ reached ESRD due to AAV progression. Median follow up duration until each event were 34.5 months, 22.8 months, and 30.5 months, respectively. Glucocorticoid was administered to $91.4 \%$ of patients and both cyclophosphamide (48.1\%) and azathioprine (47.6\%) were most commonly used as induction therapy and maintenance therapy, respectively (Table 1). 
Table 1

Characteristics of AAV patients with at diagnosis and during follow-up $(\mathrm{N}=210)$

\section{AAV patients}

Values

At the time of diagnosis

\section{Demographic data}

Age (years)

$59.0(20.3)$

Male gender $(\mathrm{N},(\%))$

$68(32.4)$

Body mass index $\left(\mathrm{kg} / \mathrm{m}^{2}\right)$

$22.2(4.4)$

Smoking history $(\mathrm{N},(\%))$

7 (3.3)

AAV Subtypes ( $N,(\%))$

MPA

$116(55.2)$

GPA

$52(24.8)$

EGPA

$42(20.0)$

ANCA positivity $(\mathrm{N},(\%))$

MPO-ANCA (or P-ANCA) positivity

$140(66.7)$

PR3-ANCA (or C-ANCA) positivity

35 (16.7)

Both ANCA positivity

9 (4.3)

ANCA negativity

$44(21.0)$

AAV-specific indices

BVAS

$12.0(11.0)$

FFS

$1.0(2.0)$

Comorbidities at diagnosis (N, (\%))

Chronic kidney disease (stage 3-5)

$60(28.6)$

Diabetes mellitus

$55(26.2)$

Hypertension

$98(46.7)$

Interstitial lung disease

$55(26.2)$

Values are expressed as a median (interquartile range, IQR) or $\mathrm{N}(\%)$.

AAV: ANCA-associated vasculitis; ANCA: antineutrophil cytoplasmic antibody; MPA: microscopic polyangiitis; GPA: granulomatosis with polyangiitis; EGPA: eosinophilic GPA; MPO: myeloperoxidase; P: perinuclear; PR3: proteinase 3; C: cytoplasmic; BVAS: Birmingham vasculitis activity score; FFS: five-factor score; ESR: erythrocyte sedimentation rate; CRP: C-reactive protein; NFI: novel fibrosis index; FCl: fibrosis cirrhosis index; ESRD: end-stage renal disease. 


\begin{tabular}{|c|c|}
\hline AAV patients & Values \\
\hline \multicolumn{2}{|l|}{ Acute-phase proteins } \\
\hline $\mathrm{ESR}(\mathrm{mm} / \mathrm{hr})$ & $60.5(70.3)$ \\
\hline $\mathrm{CRP}(\mathrm{mg} / \mathrm{L})$ & $13.2(70.6)$ \\
\hline NFI & $0.61(0.95)$ \\
\hline $\mathrm{FCl}$ & $0.032(0.037)$ \\
\hline \multicolumn{2}{|l|}{ During the follow-up period } \\
\hline Follow-up duration (months) & $34.5(64.6)$ \\
\hline \multicolumn{2}{|l|}{ Poor outcomes during follow-up (N, (\%)) } \\
\hline All-cause mortality (N, (\%)) & $21(10.0)$ \\
\hline Follow-up duration based on all-cause mortality (months) & $34.5(64.7)$ \\
\hline Relapse (N, (\%)) & $72(34.3)$ \\
\hline Follow-up duration based on relapse (months) & $22.8(44.0)$ \\
\hline ESRD (N, (\%)) & $38(18.1)$ \\
\hline Follow-up duration based on ESRD (months) & $30.5(69.4)$ \\
\hline \multicolumn{2}{|l|}{ Medications administered during follow-up (N, (\%)) } \\
\hline Glucocorticoid & $192(91.4)$ \\
\hline Cyclophosphamide & $101(48.1)$ \\
\hline Rituximab & $31(14.8)$ \\
\hline Azathioprine & $100(47.6)$ \\
\hline Mycophenolate mofetil & $23(11.0)$ \\
\hline Tacrolimus & $11(5.2)$ \\
\hline Methotrexate & $19(0.0)$ \\
\hline \multicolumn{2}{|c|}{ Values are expressed as a median (interquartile range, IQR) or $\mathrm{N}(\%)$. } \\
\hline \multicolumn{2}{|c|}{$\begin{array}{l}\text { AAV: ANCA-associated vasculitis; ANCA: antineutrophil cytoplasmic antibody; MPA: microscopic } \\
\text { polyangiitis; GPA: granulomatosis with polyangiitis; EGPA: eosinophilic GPA; MPO: myeloperoxidase; } \\
\text { P: perinuclear; PR3: proteinase 3; C: cytoplasmic; BVAS: Birmingham vasculitis activity score; FFS: } \\
\text { five-factor score; ESR: erythrocyte sedimentation rate; CRP: C-reactive protein; NFI: novel fibrosis index } \\
\text { FCl: fibrosis cirrhosis index; ESRD: end-stage renal disease. }\end{array}$} \\
\hline
\end{tabular}

\section{Correlation of continuous variables at diagnosis}


$\mathrm{NFI}$ and $\mathrm{FCl}$ tended to be correlated with the cross-sectional activity of AAV based on BVAS but it did not reach statistical significance $(r=0.133, P=0.054$ and $r=0.133, P=0.055$, respectively). Furthermore, neither NFI nor FCl was significantly correlated with age, BMI, FFS, ESR and CRP at all (Table 2). Therefore, NFI was not associated with the cross-sectional BVAS.

Table 2

Correlation of continuous variables with either $\mathrm{NFI}$ or $\mathrm{FCl}$ at diagnosis in AAV patients

\begin{tabular}{|c|c|c|c|c|}
\hline Variables & Based on NFI & & Based on $\mathrm{FCl}$ & \\
\hline & Correlation coefficient $(r)$ & $P$ value & Correlation coefficient $(r)$ & $P$ value \\
\hline Age & 0.061 & 0.380 & 0.063 & 0.366 \\
\hline BMI & 0.046 & 0.511 & 0.046 & 0.503 \\
\hline BVAS & 0.133 & 0.054 & 0.133 & 0.055 \\
\hline FFS & 0.046 & 0.508 & 0.046 & 0.504 \\
\hline ESR & 0.009 & 0.898 & 0.010 & 0.885 \\
\hline CRP & -0.013 & 0.846 & -0.005 & 0.937 \\
\hline
\end{tabular}

\section{Comparison of $\mathrm{FCl}$ and $\mathrm{NFI}$ at diagnosis based on each poor outcome}

Deceased AAV patients exhibited a higher NFI at diagnosis than survived AAV patients, but it was not statistically significant (1.26 vs. $0.59, \mathrm{P}=0.055)$. However, there were no significant differences in $\mathrm{NFI}$ at diagnosis between AAV patients with relapse and those without relapse or AAV patients with ESRD and those without ESRD. On the other hand, $\mathrm{FCl}$ at diagnosis did not differ between the three groups classified according to either all-cause mortality or relapse or ESRD occurrence (Fig. 1).

\section{Cut-offs of $\mathrm{NFI}$ and $\mathrm{FCl}$ at diagnosis for each poor outcomes}

To obtain the cut-offs of $\mathrm{NFI}$ and $\mathrm{FCl}$ at diagnosis for each poor outcome, we conducted the ROC curve analysis, but could not obtain the cut-offs. Instead, we arbitrarily defined the cut-offs of $\mathrm{NFI}$ and $\mathrm{FCl}$ as lower limit of the highest quartile at diagnosis: $\mathrm{NFI} \geq 1.24$ and $\mathrm{FCI} \geq 0.057$.

\section{Comparison of cumulative survival rates}

AAV patients with $\mathrm{NFI}$ at diagnosis $\geq 1.24$ exhibited a significantly lower cumulative patients' survival rate than those with NFI at diagnosis $<1.24(P=0.002)$. However, the cumulative relapse-free and ESRD-free 
survival rates were not different between AAV patients with NFI at diagnosis $\geq 1.24$ and those without. Meanwhile, $\mathrm{FCl}$ at diagnosis $\geq 0.057$ was unable to predict any poor outcome of AAV (Fig. 2).

\section{Cox hazards model analysis for all-cause mortality}

To clarify whether NFI at diagnosis $\geq 1.24$ might be an independent predictor of all-cause mortality during follow-up, we conducted the univariable and multivariable Cox hazards model analysis using variables at diagnosis for all-cause mortality. In the univariable analysis, age, male gender, BVAS, hypertension, interstitial lung disease, $\mathrm{CRP}$ and $\mathrm{NFI}$ at diagnosis $\geq 1.24$ were significantly associated with all-cause mortality. In the multivariable analysis, age (HR 1.049, 95\% confidence interval (CI) 1.05, 1.095), BVAS (HR $1.082,95 \% \mathrm{Cl} 1.010,1.159)$, interstitial lung disease (HR 4.728, 95\% $\mathrm{Cl} 1.878,11.901)$ and NFI at diagnosis $\geq 1.24$ (HR 2.850, 95\% Cl 1.026, 7.910) were turned out to be independent predictors of allcause mortality during follow-up in AAV patients (Table 3 ). 
Table 3

Cox hazards model analysis of variables at diagnosis for all-cause mortality during follow-up in AAV patients

\begin{tabular}{|c|c|c|c|c|c|c|}
\hline \multirow[t]{2}{*}{ Variables } & \multicolumn{3}{|c|}{ Univariable } & \multicolumn{3}{|c|}{ Multivariable } \\
\hline & HR & $95 \% \mathrm{Cl}$ & $\begin{array}{l}P \\
\text { value }\end{array}$ & HR & $95 \% \mathrm{Cl}$ & $\begin{array}{l}\mathrm{P} \\
\text { value }\end{array}$ \\
\hline Age & 1.060 & $\begin{array}{l}1.019 \\
1.104\end{array}$ & 0.004 & 1.049 & $\begin{array}{l}1.005 \\
1.095\end{array}$ & 0.029 \\
\hline Body mass index & 1.115 & $\begin{array}{l}0.962 \\
1.293\end{array}$ & 0.147 & & & \\
\hline Male gender & 2.410 & $\begin{array}{l}1.021 \\
5.690\end{array}$ & 0.045 & 2.289 & $\begin{array}{l}0.899 \\
5.830\end{array}$ & 0.082 \\
\hline Smoking history & 1.964 & $\begin{array}{l}0.262 \\
14.734\end{array}$ & 0.512 & & & \\
\hline $\begin{array}{l}\text { MPO-ANCA (or P-ANCA) } \\
\text { positivity }\end{array}$ & 1.784 & $\begin{array}{l}0.682 \\
4.664\end{array}$ & 0.238 & & & \\
\hline $\begin{array}{l}\text { PR3-ANCA (or C-ANCA) } \\
\text { positivity }\end{array}$ & 1.036 & $\begin{array}{l}0.348 \\
3.088\end{array}$ & 0.949 & & & \\
\hline BVAS & 1.081 & $\begin{array}{l}1.002 \\
1.143\end{array}$ & 0.007 & 1.082 & $\begin{array}{l}1.010 \\
1.159\end{array}$ & 0.024 \\
\hline $\begin{array}{l}\text { Chronic kidney disease (stage } \\
3-5 \text { ) }\end{array}$ & 1.985 & $\begin{array}{l}0.833 \\
4.733\end{array}$ & 0.122 & & & \\
\hline Diabetes mellitus & 1.015 & $\begin{array}{l}0.393 \\
2.619\end{array}$ & 0.976 & & & \\
\hline Hypertension & 3.132 & $\begin{array}{l}1.145 \\
8.566\end{array}$ & 0.026 & 1.414 & $\begin{array}{l}0.471 \\
4.241\end{array}$ & 0.536 \\
\hline Interstitial lung disease & 5.614 & $\begin{array}{l}2.307 \\
13.663\end{array}$ & $<.001$ & 4.728 & $\begin{array}{l}1.878 \\
11.901\end{array}$ & 0.001 \\
\hline ESR & 1.009 & $\begin{array}{l}0.998 \\
1.020\end{array}$ & 0.108 & & & \\
\hline CRP & 1.007 & $\begin{array}{l}1.001 \\
1.014\end{array}$ & 0.022 & 0.999 & $\begin{array}{l}0.991 \\
1.007\end{array}$ & 0.768 \\
\hline NFI at diagnosis $\geq 1.24$ & 3.627 & $\begin{array}{l}1.538 \\
8.556\end{array}$ & 0.003 & 2.850 & $\begin{array}{l}1.026 \\
7.910\end{array}$ & 0.044 \\
\hline \multicolumn{7}{|c|}{$\begin{array}{l}\text { AAV: ANCA-associated vasculitis; ANCA: antineutrophil cytoplasmic antibody; MPO: myeloperoxidase; } \\
\text { P: perinuclear; PR3: proteinase 3; C: cytoplasmic; BVAS: Birmingham vasculitis activity score; ESR: } \\
\text { erythrocyte sedimentation rate; CRP: C-reactive protein; NFI: novel fibrosis index. }\end{array}$} \\
\hline
\end{tabular}


When we classified AAV patients into two groups based on the calculated cut-off of NFI, 54 of 210 patients were partitioned into the group with NFI at diagnosis $\geq 1.24$. All-cause mortality was identified more frequently in AAV patients with NFI at diagnosis $\geq 1.24$ than those with NFI at diagnosis $<1.24$ ( $20.4 \%$ vs. $6.3 \%, P=0.003$ ). Furthermore, AAV patients with $\mathrm{NFI}$ at diagnosis $\geq 1.24$ had a significantly higher risk for all-cause mortality than those with NFI at diagnosis $<1.24$ (RR $3.735,95 \% \mathrm{Cl} 1.468,9.385)$ (Fig. 3).

\section{Discussion}

In this study, we investigated whether NFI, one of the indices for liver fibrosis, might be associated with the cross-sectional activity of AAV and furthermore could predict the poor outcomes, particularly all-cause mortality in immunosuppressive drug-naïve patients with AAV. We found four valuable results as follows: firstly, NFI could reflect the cross-sectional neither activity of AAV based on BVAS nor acute-phase reactants including ESR and CRP. Secondly, when the cut-off of NFI at diagnosis was defined as the lower limit of the higher quartile, AAV patients with $\mathrm{NFI}$ at diagnosis $\geq 1.24$ exhibited a significantly lower cumulative patients' survival rate than those with NFI at diagnosis $<1.24$. Thirdly, in the multivariable Cox hazards model analysis, NFI at diagnosis $\geq 1.24$ could independently predict all cause-mortality during follow-up together with age, BVAS and interstitial lung disease at diagnosis. Lastly, AAV patients with NFI at diagnosis $\geq 1.24$ had a significantly higher risk for all-cause mortality than those with NFI at diagnosis $<1.24$ (RR 3.735).

When we assessed the frequency of affected organs based on the nine items of BVAS in our study population, the most common affected item was renal manifestation $(60.0 \%)$, followed by pulmonary manifestation (57.1\%) and ear nose throat (ENT) manifestation (44.8\%). We wondered which specific organ involvement was associated with $\mathrm{NFI}$, and found that only the frequency of ENT manifestation among nine manifestations exhibited a significant difference in NFI. Ninety-four AAV patients with ENT manifestation exhibited a significantly higher median NFI than 116 AAV patients without ( 0.74 vs. $0.50, P$ $=0.024)$. So, what mechanism made NFI higher in patients with ENT manifestation compared to those without? Although it has been controversial whether sinusitis might be a typical surrogate marker for GPA or not, it is clear that sinusitis is the most common symptom of ENT involvement of AAV [13]. We conducted the literature review on the association of sinusitis with the four variables that compose a formula of NFI and found a previous study reporting that serum albumin was reported to be statistically lower in patients with chronic rhinosinusitis compared to those without [14]. However, in this study serum albumin did not differ between AAV patients with ENT manifestation and those without. Also, since serum albumin is more clinically linked to renal manifestation, it is difficult to explain the difference in NFI between AAV patients with and those without ENT manifestation by the results of this previous study alone.

The cur-off of NFI or FCl that predict the all-cause mortality was not obtained by using the ROC curve that does not include the period in the analysis. For this reason, the cut-offs of $\mathrm{NFI}$ and $\mathrm{FCl}$ at diagnosis for allcause mortality were inevitably set as the lower limit of the highest quartile for each. In the Kaplan Meier 
survival analysis, in addition to all-cause mortality which showed a statistically significant difference based on NFI at diagnosis $\geq 1.24$, ESRD also showed a trend of significance with a $P$ value of 0.1 or less $(P=0.072)$. Therefore, although statistical significance was not reached, the areas of $\mathrm{NFI}$ and $\mathrm{FCl}$ at diagnosis for all-cause mortality and ESRD using the ROC curve were qualitatively compared. First of all, in the ROC curve analysis based on all-cause mortality, $\mathrm{NFI}$ at diagnosis showed a wider area under the curve than $\mathrm{FCl}$ at diagnosis. Moreover, in the ROC curve analysis based on ESRD, NFI at diagnosis also showed a wider area under the curve than $\mathrm{FCl}$ at diagnosis (Supplementary Fig. 1). Therefore, although the statistical significance was low and the follow-up period was not taken into account, NFI at diagnosis tended to better predict the occurrence of all-cause mortality and ESRD during follow-up than $\mathrm{FCl}$ at diagnosis.

The exact mechanism of how NFI at diagnosis independently predict all-cause mortality during follow-up in AAV patients is unclear, however, we made some assumptions. Firstly, with regard to serum albumin, hypoalbuminemia is well-known major risk factor for poor prognosis including all-cause mortality and ESRD occurrence in AAV patients [15]. Since serum albumin is located in the denominator in the formula of $\mathrm{NFI}, \mathrm{NFI}$ and serum albumin theoretically show an inverse correlation. Therefore, it can be inferred that the smaller the albumin, the higher the NFI, so the highest quartile of NFI at diagnosis, which indicates the lower level of serum albumin, could predict all-cause mortality in AAV patients. The Pearson correlation analysis performed in our study data did not reach statistical significance, but showed a tendency of a negative correlation $(r=-0.119, P=0.084)$. In fact, using the Spearman correlation analysis, serum albumin was significantly and inversely correlated with NFI $\left(r^{2}=-0.322, P<0.001\right)$. These results support our assumption that NFI can predict all-cause mortality through serum albumin.

Secondly, with regard to platelet count, there have been studies demonstrating that platelet counts reflect the inflammatory burden of AAV, correlate with BVAS, and may predict the poor outcomes in AAV patients $[10,16]$. Since platelet count is also located in the denominator of the formula of NFI, theoretically, the larger the platelet counts, the smaller the NFI. However, the results from our data were not. When we focus on two variables located in the denominator, serum albumin can be considered to have a direct effect on the increase in NFI more than platelet count. Therefore, we concluded that the highest quartile of NFI at diagnosis made a more direct contribution to predicting all-cause mortality through reduced serum albumin which could reflect the high degree of inflammation and malnutrition.

In the Kaplan Meier survival analysis, the cumulative ESRD-free survival rate in AAV patients with NFI at diagnosis $\geq 1.24$ was lower than that in AAV patients with NFI at diagnosis $<1.24(P=0.072)$, although it was not statistically significant. This result may suggest that analysing a larger number of patients can yield statistically significant results.

How can NFI at diagnosis, which belongs to the highest quartile, show a tendency to predict ESRD during follow-up in AAV patients? Firstly, with regard to comorbidity of chronic kidney disease, AAV patients with chronic kidney disease at diagnosis showed higher NFI than those without $(0.86$ vs. $0.55, P=0.060)$. 
Therefore, it can be assumed that NFI reflected the accompanying chronic kidney disease at diagnosis, which could have the potential to predict ESRD occurrence in AAV patients [17].

Secondly, with regard to serum albumin, high NFI means reduced serum albumin at diagnosis as described above. Reduced serum albumin, in turn, is associated with renal manifestation and increased BVAS, and furthermore is another important risk factor for ESRD $[3,18]$. Using the univariable Cox hazards model analysis, serum albumin at diagnosis was significantly associated with ESRD during the follow-up period based on ESRD occurrence (HR 0.585, 95\% Cl 0384, 0.890). These results support our assumption that NFI can predict ESRD through serum albumin.

Thirdly, NFI is originally an index for liver fibrosis. Nevertheless, how can it predict ESRD? Although the cells involved in liver fibrosis and renal fibrosis are different, biological factors such as cytokine and chemokine that ultimately induce and promote collagen deposition in organs may share common signal pathways in the two difference fibrosis-process $[9,20]$. Therefore, although it is difficult to explain the exact mechanism, it is expected that the index of liver fibrosis can predict the process of renal fibrosis to some extent.

To our best knowledge, this is the first study to demonstrate that NFI at diagnosis could independently predict all-cause mortality in immunosuppressive drug-naïve AAV patients without chronic liver diseases. The discovery of a new predictor of all-cause mortality in AAV patients is an important advantage of our research. In addition, our study was conducted in a single institution, which may be a drawback, but it is also an advantage in that chronic liver diseases at the time of diagnosis and during the follow-up period were strictly excluded from this study. However, our study has several limitations. First, although chronic liver diseases were excluded from this study, there was no information on liver fibrosis examined by transient elastography or ultrasonography in patients with high $\mathrm{NFI}$. In particular, the disadvantage is that there was no serial information on liver fibrosis in deceased patients with AAV. Second, due to the limitations of a retrospective single institutional study, there is a possibility of selection bias, and missing data among clinical data could not be completely excluded.

\section{Conclusions}

$\mathrm{NFI}$ at diagnosis was not associated with the cross-sectional BVAS, whereas the highest quartile of NFI at diagnosis could independently predict all-cause mortality during follow-up in immunosuppressive drugnaïve AAV patients without substantial liver diseases.

\section{Abbreviations}

ANCA: antineutrophil cytoplasmic antibody; AAV: ANCA-associated vasculitis; BMI: body mass index; BVAS: Birmingham vasculitis activity score; $\mathrm{C}$ : cytoplasmic; $\mathrm{Cl}$ : confidence interval; CRP: C-reactive protein; EGPA: eosinophilic granulomatosis with polyangiitis; ENT: ear nose throat; ESR: Erythrocyte sedimentation rate; ESRD: end-stage renal disease; FCl: fibrosis cirrhosis index; FFS: five-factor score; 
GPA: granulomatosis with polyangiitis; HR: hazard ratio; IFA: immunofluorescence assay; MPA: microscopic polyangiitis; MPO: myeloperoxidase; NFI: novel fibrosis index; P: perinuclear; PR3: proteinase 3; RR: relative risk; VDI: vasculitis damage index. ;

\section{Declarations}

\section{Acknowledgement}

Not applicable

\section{Funding}

This research was supported by a grant from the Korea Health Technology R\&D Project through the Korea Health Industry Development Institute, funded by the Ministry of Health and Welfare, Republic of Korea (HI14C1324).

\section{Availability of data and materials}

The data used and analysed in this study are available from the corresponding author on reasonable request.

\section{Author's contributions}

JYP and SWL participate in research design, the writing of the final version of manuscript, and the performance of the research. JYP, SSA, LEL and SWL contributed to the acquisition of data, interpretations of data. JYP, SSA and SWL participate in the preparation of the draft manuscript. GMC conducted the statistical analyses and validate the interpretation. In particular, LEL helped with English editing. All authors read and approved the final revision of the manuscript.

\section{Ethics approval}

This study was approved by the Institutional Review Board of Severance Hospital (4-2017-0673), who waived the need for patient written informed consent, as this was a retrospective study.

\section{Consent to publication}

Not applicable.

\section{Competing interests}

The authors declare that they have no competing interests.

\section{References}


1. Jennette JC, Falk RJ, Bacon PA, Basu N, Cid MC, Ferrario F, et al. 2012 revised International Chapel Hill Consensus Conference Nomenclature of Vasculitides. Arthritis Rheum 2013;65:1-11.

2. Watts R, Lane S, Hanslik T, Hauser T, Hellmich B, Koldingsnes W, et al. Development and validation of a consensus methodology for the classification of the ANCA-associated vasculitides and polyarteritis nodosa for epidemiological studies. Ann Rheum Dis. 2007;66:222-7.

3. Mukhtyar C, Lee R, Brown D, Carruthers D, Dasgupta B, Dubey S, et al. Modification and validation of the Birmingham Vasculitis Activity Score (version 3). Ann Rheum Dis. 2009;68:1827-32.

4. Exley AR, Bacon PA, Luqmani RA, Kitas GD, Gordon C, Savage CO, et al. Arthritis Rheum. 1997;40:371-80.

5. Guillevin L, Pagnoux C, Seror R, Mahr A, Mouthon L, Le Toumelin P, et al. The Five-Factor Score revisited: assessment of prognoses of systemic necrotizing vasculitides based on the French Vasculitis Study Group (FVSG) cohort. Med (Baltim). 2011;90:19-27.

6. Willeke P, Schlüter B, Limani A, Becker H, Schotte H. Liver involvement in ANCA- associated vasculitis. Clin Rheumatol. 2016;35:387-94.

7. Park HJ, Park JY, Jung SM, Song JJ, Park YB, Lee SW. Fibrosis-4 index at diagnosis is associated with all-cause mortality in patients with microscopic polyangiitis and granulomatosis with polyangiitis. BMC Gastroenterol. 2019;19:90.

8. Kim SU, Kim BK, Park JY, Kim DY, Ahn SH, Park YB, et al. Fibrosis-4 index at diagnosis can predict allcause mortality in patients with rheumatoid arthritis: A retrospective monocentric study. Mod Rheumatol. 2020;30:70-7.

9. Hussain A, Gul MA, Khalid MU. Validation of Novel Fibrosis Index (NFI) for assessment of liver fibrosis: comparison with transient elastography (FibroScan). BMJ Open Gastroenterol. 2019;6:e000316.

10. Park PG, Yoo BW, Song JJ, Park YB, Lee SW. Will the HALP score help to assess the activity and predict the prognosis of antineutrophil cytoplasmic antibody-associated vasculitis? Clin Exp Rheumatol. 2020;38(Suppl 124):236-7.

11. Kim Y, Choi H, Jung SM, Song JJ, Park YB, Lee SW. Systemic immune- inflammation index could estimate the cross-sectional high activity and the poor outcomes in immunosuppressive drug-naïve patients with antineutrophil cytoplasmic antibody-associated vasculitis. Nephrology (Carlton). 2019;24:711-7.

12. McAdoo SP, Medjeral-Thomas N, Gopaluni S, Tanna A, Mansfield N, Galliford J, et al. Long-term follow-up of a combined rituximab and cyclophosphamide regimen in renal anti-neutrophil cytoplasm antibody-associated vasculitis. Nephrol Dial Transplant. 2019;34:63-73.

13. Kwon HC, Ahn SS, Jung SM, Song JJ, Park YB, Lee SW. Clinical implication of chronic paranasal sinusitis for the classification of microscopic polyangiitis. Int J Clin Pract. 2020;74:e13431.

14. Karataş D, Yüksel F. Albumin Levels in Patients With Chronic Rhinosinusitis With Nasal Polyp. J Craniofac Surg. 2015;26:e706-8. 
15. Xu PC, Tong ZY, Chen T, Gao S, Hu SY, Yang XW, et al. Hypoalbuminaemia in antineutrophil cytoplasmic antibody-associated vasculitis: incidence and significance. Clin Exp Rheumatol. 2018;36:603-11.

16. Park HJ, Jung SM, Song JJ, Park YB, Lee SW. Platelet to lymphocyte ratio is associated with the current activity of ANCA-associated vasculitis at diagnosis: a retrospective monocentric study. Rheumatol Int. 2018;38:1865-71.

17. Hallan SI, Coresh J, Astor BC, Asberg A, Powe NR, Romundstad S, et al. International comparison of the relationship of chronic kidney disease prevalence and ESRD risk. J Am Soc Nephrol. 2006;17:2275-84.

18. Crnogorac M, Horvatic I, Toric L, Galesic Ljubanovic D, Tisljar M, Galesic K. Clinical, serological and histological determinants of patient and renal outcome in ANCA-associated vasculitis with renal involvement: an analysis from a referral centre. Int Urol Nephrol. 2017;49:1419-31.

19. Elpek G. Cellular and molecular mechanisms in the pathogenesis of liver fibrosis: An update. World J Gastroenterol. 2014;20:7260-76.

20. Nogueira A, Pires MJ, Oliveira PA. Pathophysiological Mechanisms of Renal Fibrosis: A Review of Animal Models and Therapeutic Strategies. In Vivo. 2017;31:1-22.

\section{Figures}
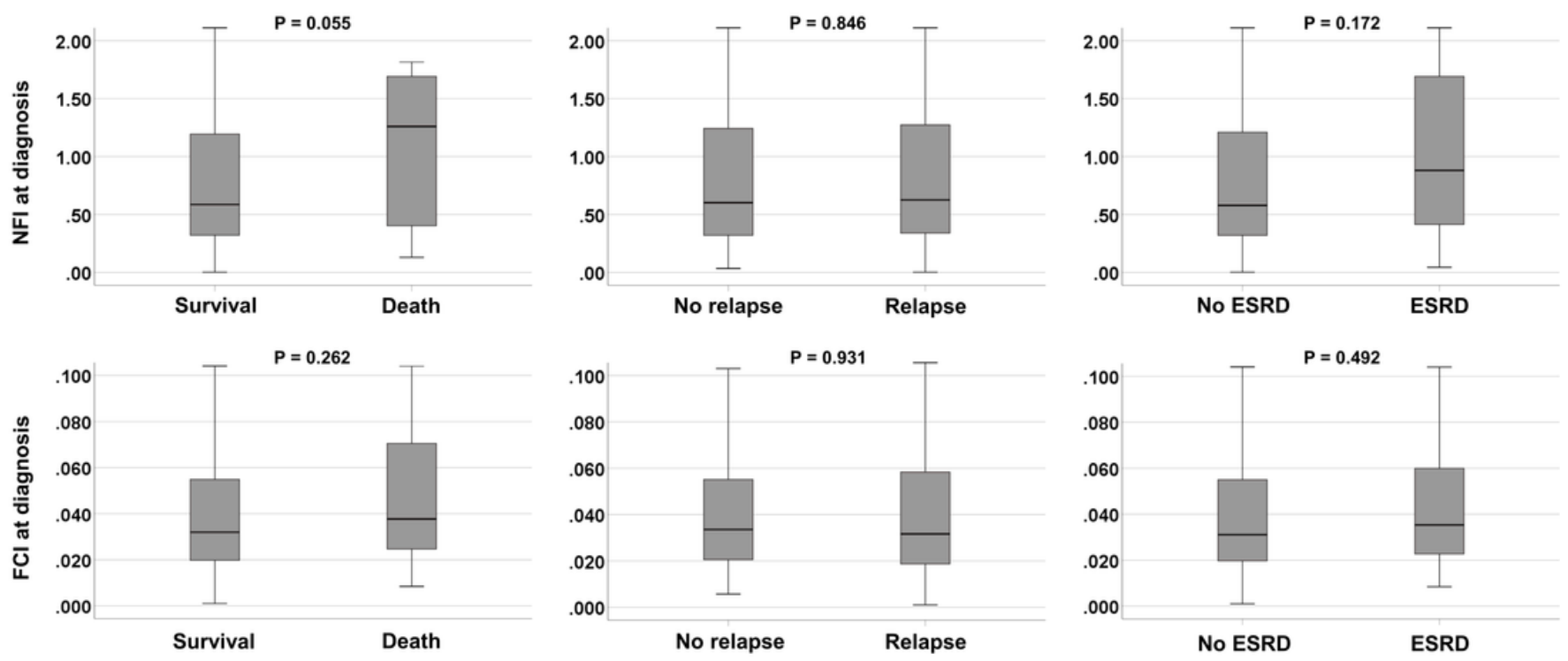

\section{Figure 1}


Comparison of $\mathrm{FCl}$ and $\mathrm{NFI}$ at diagnosis based on each poor outcome. Among six conditions, only NFI at diagnosis in deceased AAV patients tended to be increased compared to survived AAV patients but it did not reach a statistical significance. NFI: novel fibrosis index; ESRD: end-stage renal disease; FCl: fibrosis cirrhosis index.
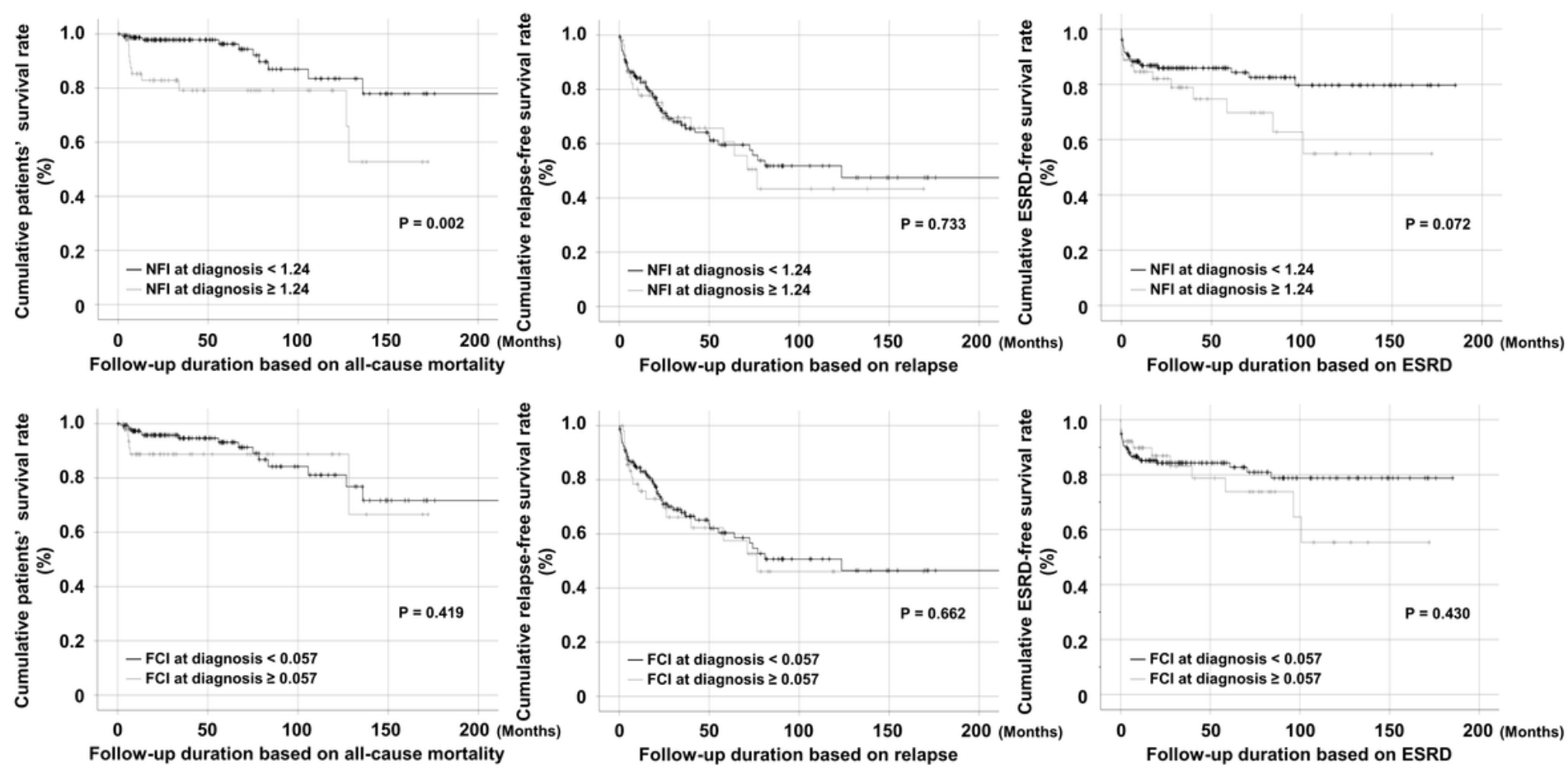

Figure 2

Comparison of the cumulative survival rates. Among six conditions, it was only found that AAV patients with $\mathrm{NFI}$ at diagnosis $\geq 1.24$ exhibited a significantly lower cumulative patients' survival rate than those without. NFI: novel fibrosis index; ESRD: end-stage renal disease; FCl: fibrosis cirrhosis index. 


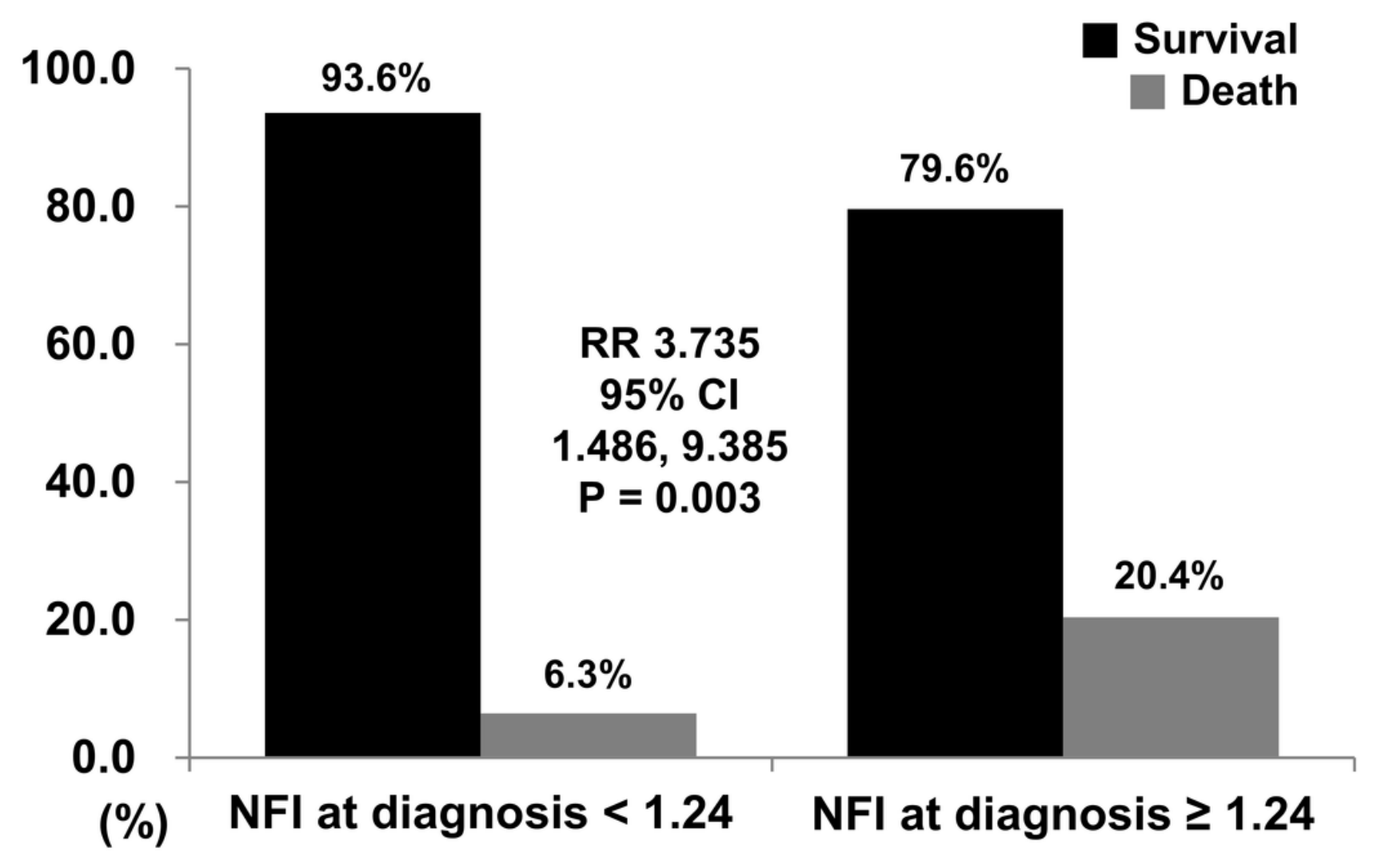

Figure 3

Relative Risk for all-cause mortality. AAV patients with NFI at diagnosis $\geq 1.24$ had a significantly higher risk for all-cause mortality than those with NFI at diagnosis < 1.24 (RR 3.735). NFI: novel fibrosis index; RR: relative risk; $\mathrm{Cl}$ : confidence interval.

\section{Supplementary Files}

This is a list of supplementary files associated with this preprint. Click to download.

- SUPPLEMENTARYFIGURE1NFIFCIAAV.tif 